\title{
Saving the universe with finite volume effects
}

\author{
Jean Alexandre ${ }^{1}$ and Katy Clough ${ }^{2}$ \\ ${ }^{1}$ Theoretical Particle Physics and Cosmology, King's College London, \\ Strand, London WC2R 2LS, United Kingdom \\ ${ }^{2}$ Astrophysics, University of Oxford, DWB, Keble Road, Oxford OX1 3RH, United Kingdom
}

(Received 7 July 2019; revised manuscript received 1 September 2019; published 15 November 2019)

\begin{abstract}
Setting aside anthropic arguments, there is no reason why the Universe should initially favor a net expanding phase rather than one experiencing a net contraction. However, a collapsing universe containing "normal" matter will end at a singularity in a finite time. We point out that there is a mechanism, derived from nonperturbative effects in quantum field theory in a finite volume, which may provide a bias toward expansion when the spacetime volume shrinks, by dynamically violating the null energy condition, without the need for modified gravity or exotic matter. We describe a scalar field component subjected to this nonperturbative effect in a cosmological background and consider its impact on a contracting phase. We discuss how this could dynamically generate the necessary initial conditions for inflation to get started, or form part of the mechanism for a nonsingular cosmological bounce.
\end{abstract}

DOI: 10.1103/PhysRevD.100.103522

\section{INTRODUCTION}

The Hawking-Penrose theorems [1,2] imply that in the absence of matter which breaks the null energy condition (NEC) $\rho+p \geq 0$, the universe, run backwards, must begin at a singularity. A corollary of this is that, under generic conditions, a collapsing universe cannot transition to a growing one, and thus if the universe started in a collapsing state, it should have ended. This statement can also be formulated in terms of the ADM decomposition [3-5], and forms the basis for work on cosmological bounces [6-8].

Such ideas are of general interest in the context of early Universe cosmology, since the Universe could in principle have started in either an expanding or contracting phase. While one can make an anthropic argument that contracting universes are simply not conducive to life, it is interesting to explore whether there are deeper, more fundamental reasons for our Universe to favor expansion.

Consider the dominant early Universe paradigm of inflation, which solves the homogeneity and isotropy problems of the Universe by postulating a period of accelerated expansion [9-12], while also providing a scale-invariant spectrum of initial perturbations. The question of how inflation actually got started is not yet fully resolved. In particular, if inflation requires "special" initial conditions in order to proceed, at least one of its key motivations may be undermined.

Numerical and analytic studies have considered the question of what initial conditions can be tolerated in a variety of different models (see Ref. [13] for a short review). In particular, recent numerical work showed that large-field models are strongly robust to initial inhomogeneities in the inflaton field [14], while small-field models are more sensitive to horizon-sized perturbations $[15,16]$. Yet these studies are open to the criticism that they bias their results by assuming that the universe begins in an expanding phase. (In Ref. [15], a mixture of expanding and contracting phases were considered, but the average expansion rate was positive.) This is a choice that must be made in setting up the initial conditions, as the first Friedmann equation is agnostic to the initial sign of $\dot{a}$. In an initially contracting universe, invoking only standard general relativity (GR) and single-field slow roll, inflation will fail, regardless of the chosen model.

In the alternative paradigm of bouncing cosmologies, the smoothing of the Universe may be achieved in a contracting phase dominated by a form of matter with equation-of-state parameter $w \gg 1$ in ekpyrotic models [6-8] or $w=1$ in the pre-big-bang scenario [17]. The generation of a scaleinvariant spectrum of perturbations is less easy to achieve, with most models invoking special mechanisms, with the exception of the matter bounce scenario [18], in which the contraction is dominated by cold matter. Clear and upto-date reviews of the topic are given in Refs. [19,20]. However, while several suggestions have been made [21], the origin of the cosmological bounce, that leads to our current expansion, is still an open question.

The requirement to break the NEC is generally considered rather exotic, apart from the well-known Casimir effect. However, consider the case where one has a secondary field which sources a negative energy density, and furthermore where the magnitude of the negative energy density grows as the spacetime volume shrinks. In the expanding case, the effect soon dilutes away. In the collapsing case, the negative energy density increases and 


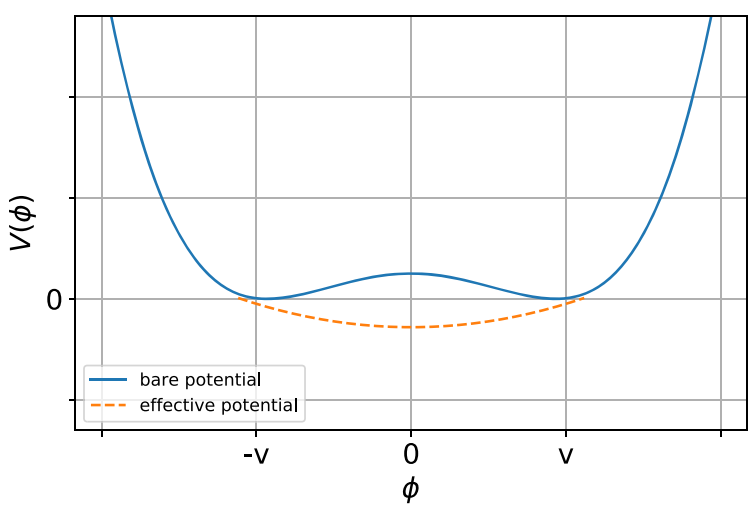

FIG. 1. Illustration of finite volume effects on a concave bare potential. Symmetry is restored by tunneling between the two bare minima, which results in a negative energy density in the ground state.

can balance the other energy contributions, slowing and eventually ending the collapse at a finite size. If, in addition, the effective equation of state violates the NEC during the collapse, it provides the necessary push for the spacetime to transition to expansion.

In this paper, we point out that such an effect is exactly that expected due to finite volume effects in a scalar field which is subject to a double-well bare potential, as illustrated in Fig. 1. Assuming the volume is smaller than some typical volume, related to the parameters of the bare potential, quantum fluctuations can tunnel between the degenerate vacua. As a consequence, the vacuum expectation value of the field vanishes and symmetry is restored. If the bare vacua have vanishing energy density, the necessity for convexity of the effective potential implies that quantum fluctuations dynamically generate a negative energy density in the ground state. The result can be generalized to higher-dimensional field spaces with a continuous set of degenerate vacua-a Higgs-like Mexican hat potential, for example - as was shown in Ref. [22] in a flat spacetime (see the Appendix for further details).

In this article, we explain why, in the context of a spatially flat Friedman-Lemaître-Robertson-Walker (FLRW) background, the magnitude of the energy density which is generated is proportional to the third inverse power of the scale factor $a^{-3}$. We also find that our "convection" field behaves as a pressureless fluid because of the nontrivial volume dependence of the effective action, and thus has the equation of state $w=0$. Since it has a negative energy density, it thus violates the NEC.

We comment on the differences between this model and the cosmological Casimir effect [23] and finish by describing a toy model in which we combine a cosmological constant with our "convection" field, in a situation where it sees the above nonperturbative convexity effect. This illustrates very simply the bias toward expansion. While such a result is known and expected for any NECviolating fluid, we emphasize that our model contains no new physics - no nonstandard couplings or modifications to the gravity sector. It is a natural dynamical consequence of the bare potential of the scalar, evolving in a cosmological spacetime.

\section{FINITE VOLUME AND THE EFFECTIVE POTENTIAL IN FLRW SPACETIME}

To define the volume in a cosmological spacetime with a FLRW metric

$$
d s^{2}=-d t^{2}+a^{2}(t)\left(d x^{2}+d y^{2}+d z^{2}\right),
$$

we assume that the flat three-dimensional space is a 3-torus with volume $V_{0}$, and is therefore periodic in comoving coordinates. The comoving volume is then

$$
V^{(3)}(t)=V_{0} a^{3}(t),
$$

and the four-dimentional volume appearing in the action is

$$
V^{(4)}=\int d^{4} x \sqrt{|g|}=V_{0} \int_{t_{i}}^{t_{f}} d t a^{3}(t),
$$

where $T \equiv t_{f}-t_{i}$ is identified with a typical time for quantum fluctuations to propagate over the volume $V_{0}$. The choice of a 3-torus (as opposed to a 3-sphere, for example) is a simplification which permits us to neglect intrinsic curvature in the spatial slice.

\section{A. Condition for convexity}

Phase transitions are generally considered in the limit of "infinite" volume, which is required for the wave functions corresponding to different degenerate vacua to be orthogonal and not overlap in field space [24]. In the case of the Higgs mechanism with vacuum $v \simeq 240 \mathrm{GeV}$, "infinite" volume means large compared to a scale proportional to $v^{-3} \simeq\left(10^{-18} \mathrm{~m}\right)^{3}$, which is obviously the case and thus ensures spontaneous symmetry breaking (SSB) in the Higgs model. For this reason, the one-particle irreducible (1PI) effective potential of a model with SSB is derived from a partial partition function, based on one vacuum only, and not on the whole field space [25].

However, in the situation where the volume is finite, tunneling between different vacua is allowed and, as a consequence, the effective potential of a scalar theory must be convex [26]: symmetry is restored by quantum fluctuations. Such a nonperturbative effect is possible only as the result of the competition between different saddle points in the partition function which defines the quantized theory [27].

We derive here the condition for tunneling between vacua to occur, and therefore for the effective action to be convex. For this, we first estimate the width, in field space, of the distribution of fluctuations over the two different homogeneous saddle points in the partition function. 
We then require this width to be of the order of the separation of these two saddle points, and thus derive a condition on the four-dimensional volume for tunneling to happen.

We are looking for an approximate condition for this tunneling effect to occur, and for clarity we therefore restrict ourselves, in this specific section, to a situation where the scale factor changes slowly compared to typical quantum fluctuations, and where flat spacetime is a good approximation. This will be especially valid in the vicinity of a cosmological bounce of an FLRW spacetime, which is the primary regime of interest.

We start with the bare potential

$$
U_{\text {bare }}(\phi)=\frac{\lambda}{24}\left(\phi^{2}-v^{2}\right)^{2}
$$

and consider the partition function for vanishing sources. We choose a vanishing energy for the vacua in order to make explicit the dynamical generation of a negative energy in the true vacuum. ${ }^{1}$ The equation of motion for the microscopic scalar field is

$$
\square \phi+\frac{\lambda}{6} \phi\left(\phi^{2}-v^{2}\right)=0,
$$

and $\phi$ is expanded above the vacua $\pm v$ as

$$
\begin{aligned}
& \phi_{1}(x)=v+\psi_{1}(x), \\
& \phi_{2}(x)=-v+\psi_{2}(x) .
\end{aligned}
$$

The semiclassical approximation described below is based on classical configurations, such that we consider here onshell fluctuations. These satisfy, for $(k=1,2)$,

$$
\square \psi_{k}+m^{2} \psi_{k}=\mathcal{O}(\lambda), \quad \text { with } \quad m^{2} \equiv \frac{\lambda}{3} v^{2},
$$

and we consider $\left(\lambda, m^{2}\right)$ as independent parameters, from which the vacuum $v$ is determined. With appropriate boundary conditions, we have then

$$
\psi_{k}(x)=\xi_{k} \sin \left(q_{\mu} x^{\mu}\right)+\mathcal{O}(\lambda), \quad \text { with } \quad q^{2}=m^{2},
$$

where $\xi_{k}$ are constant amplitudes to be integrated in the partition function. The terms $\mathcal{O}(\lambda)$ in the fluctuations $\psi_{k}$ correspond to harmonics with momenta $2 q_{\mu}$ and $3 q_{\mu}$, such that the action for these fluctuations is $(k=1,2)$

\footnotetext{
${ }^{1}$ Given that the convection field would currently occupy one of the vacua (in the large-volume limit of our present-day Universe), their energy densities must be smaller than the observed cosmological constant. For such small values, the convex part of the potential could still potentially probe negative energies, but taking them to be zero avoids the choice of a finely tuned value. This could also be motivated, for example, by an underlying supersymmetric model which fixes their value to zero.
}

$$
\begin{aligned}
S_{k}= & \frac{1}{2} \int d^{4} x\left(q^{2} \xi_{k}^{2} \cos ^{2}\left(q_{\mu} x^{\mu}\right)-m^{2} \xi_{k}^{2} \sin ^{2}\left(q_{\mu} x^{\nu}\right)\right. \\
& \left.-\frac{\lambda}{12} \xi_{k}^{4} \sin ^{4}\left(q_{\mu} x^{\mu}\right)+\mathcal{O}\left(\lambda^{2}\right)\right) \\
= & -\frac{\lambda}{64} \xi_{k}^{4} V^{(4)}+\mathcal{O}\left(\lambda^{2}\right) .
\end{aligned}
$$

In the partition function, the latter actions lead to distributions peaked over the vacua $\pm v$, with half-width

$$
\Delta=\left(\frac{64}{\lambda V^{(4)}}\right)^{1 / 4}
$$

For these distributions to overlap considerably in field space, and therefore for tunneling between vacua to occur, this half-width should be at least of order $v$, which leads to the convexity condition

$$
\lambda v^{4} V^{(4)} \lesssim 64
$$

This bound is quite conservative-tunneling would occur beyond it, but at a slower rate.

\section{B. Wilsonian approach}

The disappearance of the concave part of the potential has been studied in the Wilsonian context [28], which in spirit is closer to the effective field theory context than the 1PI potential, and therefore is more commonly used in the framework of cosmology. The Wilsonian effective potential is flat between the bare vacua (and therefore convex), which corresponds to the so-called Maxwell construction [29], by analogy with the transition from liquid to vapor, when studied with the Van de Waals equation of state. ${ }^{2}$ How is this consistent with symmetry restoration? In a flat effective potential, the field is equally likely to be found at any point in the flat section, and thus it describes the coexistence of different phases, each with different ground states, which on average give a symmetric ground state. As the volume tends to infinity, tunneling is suppressed, and bubbles of each phase nucleate, such that at any localized point in space one need only consider the potential around the relevant vacuum for that phase. It is in this large-volume limit that one usually considers the potential for the inflaton, for example.

In this work, we consider the 1PI effective potential, since unlike the Wilsonian one, it takes into account finite volume effects and leads to symmetry restoration locally, not just on average. The 1PI effective potential is identical to the Wilsonian effective potential for infinite volume only.

\footnotetext{
${ }^{2}$ Note that the Maxwell construction has been used to describe spinodal inflation [30], which is different from the mechanism we describe here.
} 


\section{Effective potential}

If we assume a regime where the volume satisfies the condition in Eq. (11), the partition function is dominated by both saddle points corresponding to the two vacua. The semiclassical approximation for the effective action is then described in Ref. [22], and we follow the same steps, also valid for curved space time, in the Appendix. The convex effective action, initially derived in Ref. [22], is

$$
\begin{aligned}
S_{\text {eff }}\left[\phi_{c}\right]= & -K+\frac{4 A}{1+8 A}\left(\frac{\phi_{c}}{v}\right)^{2} \\
& +\frac{8 A\left(-3+12 A+128 A^{3}\right)}{3(1+8 A)^{4}}\left(\frac{\phi_{c}}{v}\right)^{4} \\
& +\mathcal{O}\left(\phi_{c} / v\right)^{6},
\end{aligned}
$$

where $K$ is a constant, $\phi_{c}$ is the homogeneous expectation value of the convection, and

$$
A \equiv \frac{\lambda}{24} v^{4} V^{(4)} .
$$

We note the following points:

(1) Equation (12) is only valid for $\left|\phi_{c}\right| \ll v$. As explained in the Appendix, the semiclassical approximation for $\left|\phi_{c}\right| \gg v$ leads to an effective action which is identical to the bare action.

(2) Given the expression for $A$, one can see that the convexity condition Eq. (11) is satisfied for $A \lesssim 8 / 3$.

(3) In addition to being convex, one can see that the effective action has a nontrivial volume dependence, and is not extensive. The effective potential, obtained after dividing by $V^{(4)}$, therefore depends on the volume in a nontrivial way.

(4) The constant $K$ is determined from continuity of the effective action [Eq. (12)] at $\phi_{c}= \pm v$, which leads to

$$
K \simeq \frac{4 A}{1+8 A}+\frac{8 A}{3(1+8 A)^{4}}\left(-3+12 A+128 A^{3}\right) .
$$

For $A \gtrsim 1$, Eq. (14) leads to

$$
K \simeq \frac{7}{12}-\frac{5}{48 A}+\frac{11}{384 A^{2}}+\cdots .
$$

In the large- $A$ limit, the resummation of all the powers of $\phi_{c} / v$ was calculated in Ref. [31] and leads to

$$
\begin{aligned}
S_{\mathrm{eff}}^{A \gg 1}\left[\phi_{c}\right]= & -\ln 2+\frac{1}{2}\left(1-\frac{\phi_{c}}{v}\right) \ln \left(1-\frac{\phi_{c}}{v}\right) \\
& +\frac{1}{2}\left(1+\frac{\phi_{c}}{v}\right) \ln \left(1+\frac{\phi_{c}}{v}\right) .
\end{aligned}
$$

This expression is independent of $A$ and is therefore intensive. Although this feature is valid for large $A$, one can see from Eq. (15) that $K$ is already approximately volume-independent if $A$ is of order 1, and therefore within the convexity regime described by Eq. (11).

The effective action in this intensive regime should not depend on the scale factor $a(t)$, and its value in the vacuum can therefore be written

$$
S_{\text {eff }}[0]=-K=-\int d^{4} x \sqrt{|g|} \frac{\rho_{0}}{a^{3}(t)},
$$

where $\rho_{0}$ is a positive constant. In the present semiclassical approximation we have

$$
\rho_{0}=\frac{K}{V_{0} T} .
$$

A more accurate value would require a better approximation for the effective action in the region $\left|\phi_{c}\right| \simeq v$. One expects then that $\rho_{0}$ depends on the parameters appearing in the bare potential, but at this stage we leave $\rho_{0}>0$ as a free parameter. The energy density $\rho_{c}$ and the pressure $p_{c}$ in the vacuum state $\phi_{c}=0$ are finally

$$
\begin{aligned}
& \rho_{c}=\left.\frac{2}{\sqrt{|g|}} \frac{\delta S_{\text {eff }}}{\delta\left|g_{00}\right|}\right|_{\phi_{c}=0}=-\frac{\rho_{0}}{a^{3}(t)}<0, \\
& p_{c}=\left.\frac{2}{\sqrt{|g|}} \frac{\delta S_{\text {eff }}}{\delta\left|g_{11}\right|}\right|_{\phi_{c}=0}=0
\end{aligned}
$$

such that the NEC is violated in the ground state, since $p_{c}+\rho_{c}<0$. Note that this vacuum state consistently satisfies the continuity equation for a fluid $\dot{\rho}_{c}=-3 H\left(\rho_{c}+p_{c}\right)$.

We highlight that the effect occurs in the range $1 \lesssim A \lesssim$ $8 / 3$, which, in the context of a cosmological collapse scenario, assumes a specific period for which the volume is the appropriate size and the effect "turns on." As discussed above, the upper bound is conservative, and the overlap of wave functions corresponding to the different vacua remains non-negligible for larger values for $A$. An interesting question, left for future work, is how this range is probed in a dynamical situation.

\section{Casimir effect}

We end this section with comments regarding the Casimir effect, which could potentially have a role for small volumes. The study of the Casimir effect in the context of cosmology was introduced in Ref. [23], where a massless scalar field in a 3-torus has a repulsive Casimir effect, and therefore contributes to an expansion of the Universe. More generally, the Casimir effect is either repulsive or attractive, depending on the coupling of the scalar field to the metric, but also on the 3-space geometry/ topology (see Ref. [32] for a review). A massless scalar 
field conformally coupled to the metric, and experiencing the Casimir effect, has an energy density $\rho$ and a pressure $p$ which scale as

$$
\rho \propto \frac{ \pm 1}{a^{4}(t)} \quad \text { and } \quad p=\frac{\rho}{3}
$$

and which could potentially destabilize the tunneling effect for small values of the scale factor, since the convection energy density scales as $a^{-3}(t)$. Nevertheless, for a massive scalar field, the energy density (20) is suppressed as [32]

$$
|\rho| \propto \frac{\left(m l_{0}\right)^{5 / 2}}{a^{3 / 2}(t)} e^{-m l_{0} a(t)}, \quad \text { with } \quad m l_{0} a(t) \gg 1
$$

where $l_{0}^{3} \sim V_{0}$. Hence, one can expect that tunneling effects remain dominant, in a certain region of parameter space at least.

The Casimir energy density may be attractive or repulsive, depending on the couplings of the scalar field to the metric and the geometry of the 3-space [33], so there are specific conditions such that the Casimir effect is repulsive. We note that the quantum corrections arising from our mechanism always have the same sign, independently of the geometry/topology of the 3-space-convexity of the potential requires that they always lead to an energy density which is lower than the one at the degenerate bare minima.

\section{COSMOLOGICAL ILLUSTRATION}

We now describe a toy cosmological model as an illustration of how the above dynamical mechanism could work to favor expansion. We consider the FLRW metric [Eq. (1)] with Hubble constant $H=\dot{a} / a$, and we assume that the matter content is dominated by two components, both of which satisfy the continuity equation $\dot{\rho}=-3 H(\rho+p)$ individually:

(1) A cosmological constant, with energy density $\rho_{f}$ and pressure $p_{f}=-\rho_{f}$. (This could be a scalar field slow-rolling in a convex potential.)

(2) The "convection" field, with a vanishing expectation value $\phi_{c}=0$, and an energy density and pressure given by the expressions in Eq. (19).

Using the Friedmann equations

$$
\begin{aligned}
H^{2} & =\frac{\kappa}{3}\left(\rho_{f}+\rho_{c}\right), \\
\dot{H}+H^{2} & =-\frac{\kappa}{6}\left(\rho_{f}+\rho_{c}+3 p_{f}+3 p_{c}\right),
\end{aligned}
$$

where $\kappa \equiv 8 \pi G$, the evolution equation is

$$
\dot{H}=\frac{\kappa}{2} \frac{\rho_{0}}{a^{3}}>0,
$$

to be solved with initial values $a_{i}=1$ and $H_{i}$ which satisfies the constraint

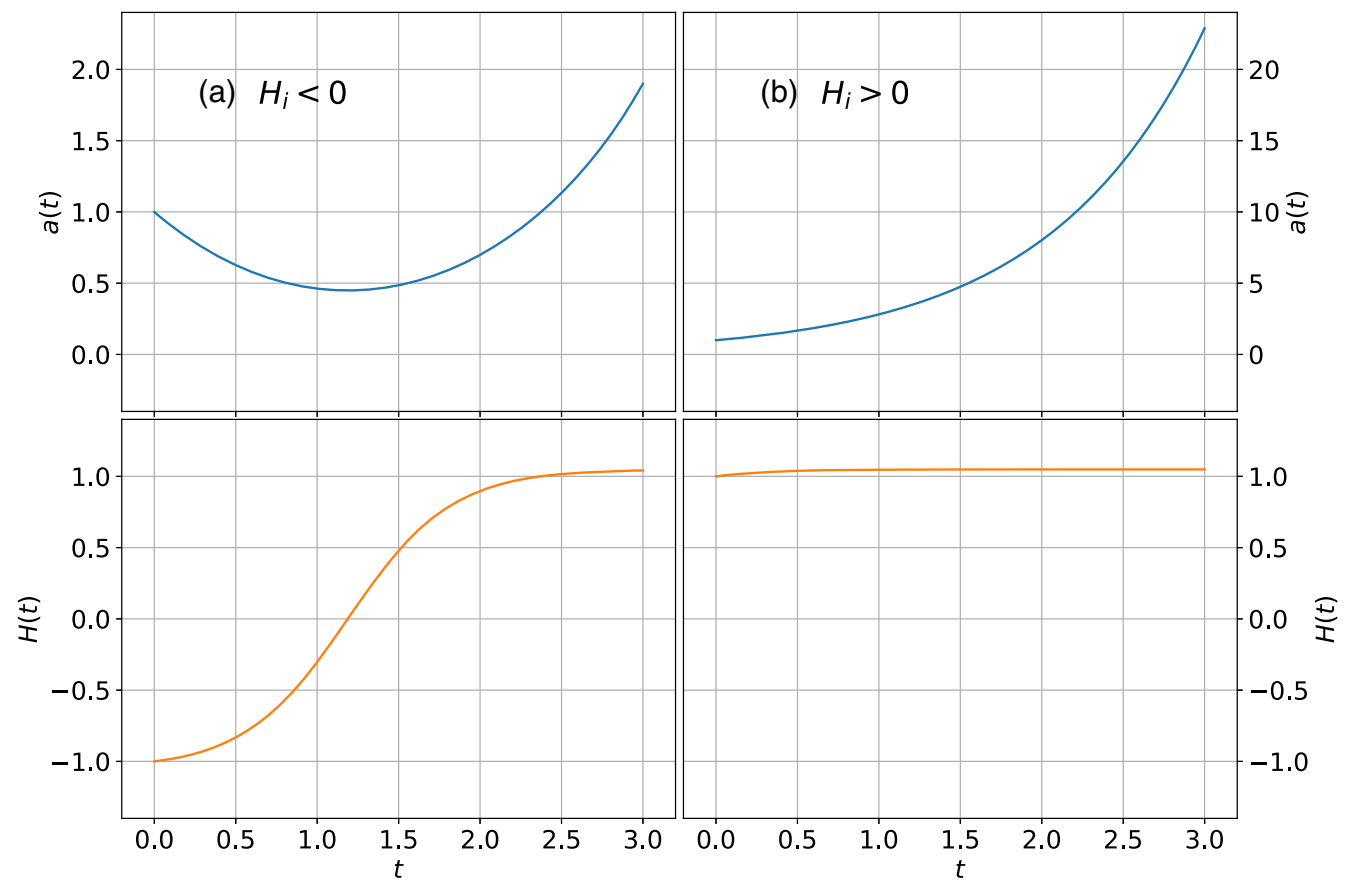

FIG. 2. An illustration of the evolution of the scale factor $a$ and Hubble constant $H$ as a function of time $t$ for the cases of (a) initial contraction and (b) initial expansion in our toy model. We see in the collapsing case that the convection field provides the necessary bounce to transition to a period of exponential expansion. If the initial condition is already expanding, the effect of the convection is quickly diluted away. 


$$
H_{i}^{2}=\frac{\kappa}{3}\left(\rho_{f}-\rho_{0}\right)
$$

The resulting evolution is shown in Fig. 2 for the cases of initially positive and negative $H$. It is clear from Eq. (23) that for our chosen components the NEC is violated, and thus the acceleration of the scale factor $\ddot{a}$ is positive, providing the necessary bounce to expansion in the contracting case.

\section{CONCLUSIONS}

In this article we have suggested a connection between finite volume effects in quantum field theory and early Universe cosmology, to answer the question "Why is the Universe expanding?" We have postulated the existence of a "convection" field which sees different degenerate bare vacua. Quantum tunneling effects result in a pressureless fluid with negative energy density, which plays a role at small volumes, but dilutes away during an expanding period. Such a fluid breaks the NEC, and thus provides a mechanism for a transition from contraction to an expanding phase. Unlike other proposed mechanisms, this does not involve nonstandard couplings for the field or a modification of the gravity sector, and thus does not result in ghosts-the dynamical mechanism is valid for any standard scalar field which can tunnel between degenerate vacua.

One naturally asks about the regime in which this effect is valid. As discussed in the text, convexity, and thus a negative energy density, is achieved for any fourdimensional volume small enough to satisfy the condition in Eq. (11), which leads to a condition $A \lesssim 8 / 3$. However, an intensive effective action (which provides the $w=0$ equation of state) is valid for $A \gtrsim 1$, which sets a minimum volume via Eq. (13). The mechanism we describe here is therefore valid within a regime where the volume is not "too big," but also not "too small." Of course, at some very small volume, quantum gravity effects should also play a role, and our description will break down. Similar nonperturbative effects have been discussed in Refs. [34,35], which take into account finite volume effects in a semiclassical description of quantum gravity. There the authors discuss the contribution of two different saddle points in the path integral for gravity, and they derive a minimum volume in order to decouple these saddle points and therefore have a well-defined cosmological evolution. For gravity to be classical, as it is in our work, the minimum volume we consider must be greater than the minimum volume defined in Refs. [34,35]. In this way, quantization of gravity would not affect the results in our regime of validity. Given that there are free parameters in both models, this inequality can easily be satisfied for a large range of parameter space.

Much work remains to be done to elucidate this interesting effect. First, one must justify the interpretation of equilibrium field theory in a dynamical spacetime.
One should also investigate what the convexity condition [Eq. (11)] means for the separation of ultraviolet modes of the convection from its infrared description in terms of a vacuum expectation value.

A better approximation for the calculation of the partition function, including nonuniform saddle points and quantum fluctuations above each saddle point, should also be made. This is quite an involved calculation, but we would expect any modifications to be relevant only away from the vacuum state which we consider here.

Finally, we have considered only the coexistence of the convection with a cosmological constant. Matter- or radiation-like components would dominate over the convection during a collapsing period, and in the present simplified description one must make the strong assumption that they are not present. Further studies are necessary to understand the interplay between the convection and different matter components, once kinetic terms are included. The latter might play an important role, depending on the flatness of the potential around the true minimum. Related to this, one would ultimately like to study inhomogeneous initial conditions in the background metric, especially since initial anisotropies tend to grow and dominate during a collapsing phase.

\section{ACKNOWLEDGMENTS}

We are grateful to Eugene Lim for his insightful comments on an earlier draft. The work of J. A. is partially supported by STFC (Grant No. ST/P000258/1). K. C. has received funding from the European Research Council (ERC) under the European Union's Horizon 2020 research and innovation program (Grant Agreement No. 693024).

\section{APPENDIX: SEMICLASSICAL APPROXIMATION}

We derive here the effective action for the bare action

$$
S[\phi]=\int d^{4} x \sqrt{|g|}\left(\frac{g_{\mu \nu}}{2} \partial^{\mu} \phi \partial^{\nu} \phi-U_{\text {bare }}(\phi)\right),
$$

with

$$
U_{\text {bare }}(\phi)=\frac{\lambda}{24}\left(\phi^{2}-v^{2}\right)^{2},
$$

when both vacua are taken into account in the partition function

$$
Z[j]=\int \mathcal{D}[\phi] \exp \left(-S[\phi]-\int d^{4} x \sqrt{|g|} j \phi\right) .
$$

The source $j$ is chosen to be constant, since we are interested in the effective potential only. The semiclassical approximation corresponds to replacing the path integral with the sum over the relevant saddle points, and neglecting quantum fluctuations above the latter. Taking into account 
the source term, the bare vacua are not degenerate, and the Coleman configuration, which asymptotically reaches the two different vacua [36], should in principle play a role. Nevertheless, as we will show, the ground state of the system consists of a vanishing background field $\phi_{c}=0$, which corresponds to a vanishing source $j$, for which this configuration does not contribute. If we focus on the background field satisfying $\left|\phi_{c}\right| \ll v$, or equivalently $|j| \ll j_{c}$, it is therefore enough to consider uniform saddlepoint configurations, and we follow here similar steps as those described in Ref. [22].

These uniform saddle points are solutions of the equation

$$
\frac{d U_{\mathrm{bare}}}{d \phi}+j=0
$$

and depend on the amplitude of the source $j$ :

(1) If $|j|>j_{c}$, where $j_{c}=\lambda v^{3} /(9 \sqrt{3})$, then Eq. (A3) has one real solution, $\phi_{0}$.

(2) If $|j|<j_{c}$, then Eq. (A3) has three real solutions, one of which is a maximum and two others which are the local minima relevant for the partition function:

$$
\begin{aligned}
& \phi_{1}=\frac{2 v}{\sqrt{3}} \cos \left[\pi / 3-(1 / 3) \arccos \left(j / j_{c}\right)\right], \\
& \phi_{2}=\frac{2 v}{\sqrt{3}} \cos \left[\pi-(1 / 3) \arccos \left(j / j_{c}\right)\right] .
\end{aligned}
$$

We consider below these two situations separately. We note that functional derivatives with respect to a uniform source $j$ are replaced by partial derivatives with respect to $V^{(4)} j$, and the background field is therefore

$$
\phi_{c} \equiv \frac{-1}{Z V^{(4)}} \frac{\partial Z}{\partial j},
$$

which is used below.

\section{Case $|j| \gg j_{c}$}

This situation describes the region $\left|\phi_{c}\right| \gg v$, where the semiclassical approximation for the partition function is

$$
Z[j] \simeq \exp \left(-\int d^{4} x \sqrt{|g|}\left(U_{\text {bare }}\left(\phi_{0}\right)+j \phi_{0}\right)\right)
$$

The background field [Eq. (A5)] is then, in this approximation,

$$
\phi_{c}=\left(U_{\mathrm{bare}}^{\prime}\left(\phi_{0}\right)+j\right) \frac{\partial \phi_{0}}{\partial j}+\phi_{0}=\phi_{0} .
$$

The semiclassical 1PI effective action is then defined by the Legendre transform

$$
\begin{gathered}
S_{\text {eff }}\left[\phi_{c}\right]=-\ln Z[j]-V^{(4)} j \phi_{c} \\
=V^{(4)} U_{\text {bare }}\left(\phi_{c}\right),
\end{gathered}
$$

leading to the semiclassical effective potential

$$
U_{\mathrm{sc}}\left(\phi_{c}\right)=U_{\text {bare }}\left(\phi_{c}\right) .
$$

The semiclassical approximation therefore does not modify the bare potential for $\left|\phi_{c}\right| \gg v$.

\section{Case $|j| \ll j_{c}$}

This situation describes the region $\left|\phi_{c}\right| \ll v$, where the two saddle points $\phi_{1}, \phi_{2}$ dominate the partition function. The latter is

$$
Z[j] \simeq \sum_{k=1}^{2} \exp \left(-\int d^{4} x \sqrt{|g|}\left(U_{\text {bare }}\left(\phi_{k}\right)+j \phi_{k}\right)\right),
$$

and the background field $\phi_{c}$ can be obtained as a Taylor expansion in $j$. This expansion can then be inverted to give the source $j$ as a Taylor expansion in $\phi_{c}$, and the 1PI effective action can then be obtained by integrating the equation of motion

$$
\frac{1}{V^{(4)}} \frac{\partial \Gamma}{\partial \phi_{c}}=-j
$$

These expansions have been made in Ref. [22], and they lead to the semiclassical convex effective action

$$
\begin{aligned}
S_{\mathrm{eff}}\left[\phi_{c}\right]= & -K+\frac{4 A}{1+8 A}\left(\frac{\phi_{c}}{v}\right)^{2} \\
& +\frac{8 A\left(-3+12 A+128 A^{3}\right)}{3(1+8 A)^{4}}\left(\frac{\phi_{c}}{v}\right)^{4} \\
& +\mathcal{O}\left(\phi_{c} / v\right)^{6},
\end{aligned}
$$

where $K$ is a constant and

$$
A \equiv \frac{\lambda}{24} v^{4} V^{(4)}
$$

We note that Ref. [22] is done in flat spacetime, where the constant $K$ has no physical consequence. In the present case, this constant is determined by $S_{\text {eff }}[ \pm v]=0$, as previously discussed in the article.

Finally, we note that the generalization of this semiclassical approximation to $O(N)$ symmetry is also derived in Ref. [22], in terms of the radial field component $r_{c}=\sqrt{\vec{\phi}_{c} \cdot \vec{\phi}_{c}}$, and leads to

$S_{\text {eff }}^{N}\left[r_{c}\right]=-K_{N}+a_{2}\left(\frac{r_{c}}{v}\right)^{2}+a_{4}\left(\frac{r_{c}}{v}\right)^{4}+\mathcal{O}\left(r_{c}^{6}\right)$, 
where $K_{N}$ is a constant and

$$
\begin{aligned}
& a_{2}=\frac{4 N A}{(1+8 A)}, \\
& a_{4}=\frac{8 N^{2} A[-3 N+2(1+5 N) A]}{(N+2)(1+8 A)^{4}}+\frac{8 N^{2} A\left[32(1-N) A^{2}+128 A^{3}\right]}{(N+2)(1+8 A)^{4}} .
\end{aligned}
$$

[1] A. Borde and A. Vilenkin, Phys. Rev. Lett. 72, 3305 (1994).

[2] S. W. Hawking and R. Penrose, Proc. R. Soc. A 314, 529 (1970).

[3] J. D. Barrow and F. J. Tipler, Mon. Not. R. Astron. Soc. 216, 395 (1985).

[4] R. L. Arnowitt, S. Deser, and C. W. Misner, Phys. Rev. 117, 1595 (1960).

[5] M. Kleban and L. Senatore, J. Cosmol. Astropart. Phys. 10 (2016) 022.

[6] P. J. Steinhardt and N. Turok, Phys. Rev. D 65, 126003 (2002).

[7] J. Khoury, B. A. Ovrut, N. Seiberg, P. J. Steinhardt, and N. Turok, Phys. Rev. D 65, 086007 (2002).

[8] J. Khoury, B. A. Ovrut, P. J. Steinhardt, and N. Turok, Phys. Rev. D 64, 123522 (2001).

[9] A. H. Guth, Phys. Rev. D 23, 347 (1981).

[10] A. D. Linde, Phys. Lett. 108B, 389 (1982).

[11] A. Albrecht and P. J. Steinhardt, Phys. Rev. Lett. 48, 1220 (1982).

[12] A. A. Starobinsky, Phys. Lett. 91B, 99 (1980).

[13] R. Brandenberger, Int. J. Mod. Phys. D 26, 1740002 (2017).

[14] W.E. East, M. Kleban, A. Linde, and L. Senatore, J. Cosmol. Astropart. Phys. 09 (2016) 010.

[15] K. Clough, R. Flauger, and E. A. Lim, J. Cosmol. Astropart. Phys. 05 (2018) 065.

[16] K. Clough, E. A. Lim, B. S. DiNunno, W. Fischler, R. Flauger, and S. Paban, J. Cosmol. Astropart. Phys. 09 (2017) 025.

[17] M. Gasperini and G. Veneziano, Astropart. Phys. 1, 317 (1993).

[18] F. Finelli and R. Brandenberger, Phys. Rev. D 65, 103522 (2002).

[19] A. Ijjas and P. J. Steinhardt, Classical Quantum Gravity 35, 135004 (2018).

[20] R. Brandenberger and P. Peter, Found. Phys. 47, 797 (2017).

[21] P. Peter and N. Pinto-Neto, Phys. Rev. D 66, 063509 (2002); T. Biswas, A. Mazumdar, and W. Siegel, J. Cosmol. Astropart. Phys. 03 (2006) 009; T. Biswas, R. Brandenberger, A. Mazumdar, and W. Siegel, J. Cosmol. Astropart. Phys. 12 (2007) 011; C. Kounnas, H. Partouche, and N. Toumbas, Classical Quantum Gravity 29, 095014 (2012).

[22] J. Alexandre and A. Tsapalis, Phys. Rev. D 87, 025028 (2013).

[23] Y. B. Zeldovich and A. A. Starobinsky, Sov. Astron. Lett. 10, 135 (1984).

[24] V. A. Miransky, Dynamical Symmetry Breaking in Quantum Field Theories (World Scientific, Singapore, 1993), p. 533.

[25] A. D. Plascencia and C. Tamarit, J. High Energy Phys. 10 (2016) 099.

[26] K. Symanzik, Commun. Math. Phys. 16, 48 (1970); J. Iliopoulos, C. Itzykson, and A. Martin, Rev. Mod. Phys. 47, 165 (1975); R. W. Haymaker and J. Perez-Mercader, Phys. Rev. D 27, 1948 (1983).

[27] S. R. Coleman, R. Jackiw, and H. D. Politzer, Phys. Rev. D 10, 2491 (1974); Y. Fujimoto, L. O'Raifeartaigh, and G. Parravicini, Nucl. Phys. B212, 268 (1983); R. J. Rivers, Z. Phys. C 22, 137 (1984).

[28] C. Wetterich, Nucl. Phys. B352, 529 (1991); J. Alexandre, V. Branchina, and J. Polonyi, Phys. Lett. B 445, 351 (1999).

[29] C. M. Bender and F. Cooper, Nucl. Phys. B224, 403 (1983); F. Cooper and B. Freedman, Nucl. Phys. B239, 459 (1984); P. Millington and P. M. Saffin, arXiv:1905.09674.

[30] D. Boyanovsky, H. J. de Vega, R. Holman, and J. F. J. Salgado, Phys. Rev. D 54, 7570 (1996); D. Boyanovsky, D. Cormier, H. J. de Vega, R. Holman, A. Singh, and M. Srednicki, arXiv:hep-ph/9609527; D. Cormier and R. Holman, Phys. Rev. D 62, 023520 (2000); S. Tsujikawa and T. Torii, Phys. Rev. D 62, 043505 (2000).

[31] J. Alexandre, Nucl. Phys. B910, 868 (2016).

[32] M. Bordag, U. Mohideen, and V. M. Mostepanenko, Phys. Rep. 353, 1 (2001).

[33] C. A. R. Herdeiro and M. Sampaio, Classical Quantum Gravity 23, 473 (2006).

[34] A. Di Tucci, J. Feldbrugge, J. L. Lehners, and N. Turok, Phys. Rev. D 100, 063517 (2019).

[35] S. F. Bramberger, A. Di Tucci, and J. L. Lehners, arXiv: 1907.05782.

[36] S. R. Coleman, Phys. Rev. D 15, 2929 (1977); 16, 1248(E) (1977); C. G. Callan, Jr. and S. R. Coleman, Phys. Rev. D 16, 1762 (1977). 